\title{
En Colombie-Britannique désormais, École catholique ou École française?
}

\section{Thomas-Marie Landry}

Volume 33, 1966

URI : https://id.erudit.org/iderudit/1007323ar

DOI : https://doi.org/10.7202/1007323ar

Aller au sommaire du numéro

Éditeur(s)

Les Éditions Historia Ecclesiæ Catholicæ Canadensis Inc.

ISSN

0318-6172 (imprimé)

1927-7067 (numérique)

Découvrir la revue

Citer cet article

Landry, T.-M. (1966). En Colombie-Britannique désormais, École catholique ou École française? Sessions d'étude - Société canadienne d'histoire de l'Église catholique, 33, 67-74. https://doi.org/10.7202/1007323ar

Tous droits réservés @ Les Éditions Historia Ecclesiæ Catholicæ Canadensis Inc., 1967
Ce document est protégé par la loi sur le droit d'auteur. L'utilisation des services d'Érudit (y compris la reproduction) est assujettie à sa politique d'utilisation que vous pouvez consulter en ligne.

https://apropos.erudit.org/fr/usagers/politique-dutilisation/ 


\title{
En Colombie-Britannique désormais, Ecole catholique ou Ecole française?
}

\author{
Communication présentée par le R.P. Thomas- \\ M. Landry, o.p., directeur des Relations exté- \\ rieures au Conseil de la Vie française en \\ Amérique, au $33^{\circ}$ Congrès annuel de la \\ Société canadienne d'Histoire de l'Eglise ca- \\ tholique, tenu à l'Université de Sherbrooke, \\ Sherbrooke, P.Q., les 11 et 12 juin 1966.
}

Les Canadiens français de la Colombie-Britannique sont des gens très sympathiques et très intéressants. Je les aime beaucoup. J'ai eu l'occasion et la joie de les fréquenter assez assidûment depuis quelques années. Je crois avoir compris un peu les très graves difficultés qui assaillent leur vie catholique, surtout leur vie française.

Ils ont, entre autres problèmes, un problème scolaire urgent, lancinant, permanent et jusqu'à tout récemment à peu près insoluble. Mais voici que, depuis quelques années, ils s'efforcent résolument de briser le carcan de fer qui semblait devoir arrêter, sinon étouffer à tout jamais leur volonté d'améliorer leurs institutions scolaires pour que celles-ci permettent à leurs enfants d'aboutir enfin à une culture française convenable. Ils sont à la veille de poser des gestes et, peut-être, d'obtenir du gouvernement de leur province des amendements à la loi scolaire qui changeront d'une manière radicale l'état d'infériorité où leurs écoles se débattent présentement. En somme, les Canadiens français de la ColombieBritannique sont en train d'écrire une page d'histoire nouvelle de la vie française en ce pays. Cette histoire, je voudrais vous la raconter cet après-midi avec autant de précision et de clarté que possible. Ceci dans l'espoir que vous vous intéressiez vous-mêmes désormais à ces amis et compatriotes de par-delà les Rocheuses canadiennes et que vous suiviez de près les péripéties de ce drame passionnant qu'ils viennent d'amorcer.

Evénements qui touchent de très près à la vie de l'Eglise en même temps, puisque sans renoncer, loin de là, à leur catholicisme ni pour eux ni pour leurs enfants, les chefs canadiens-français de là-bas, laïques sans doute, mais prêtres aussi, religieux et religieuses, en sont arrivés à la conviction que l'école confessionnelle actuelle, malgré ses très grands mérites, ne peut assurer la survie, encore moins la pleine vie française, à la jeune génération d'aujourd'hui, moins encore à celle de demain et que, sous peine de mourir en tant que minorité française en ColombieBritannique, il faut, dans le plus bref délai possible, une nouvelle formule scolaire, dont l'ingrédient de base soit non plus la confessionnalité, donc pour eux le catholicisme, comme ce fut toujours le cas jusqu'ici, mais plutôt la langue et la culture françaises. Par où l'on voit jusqu'à quel point l'Eglise se trouve ici concernée, puisque c'est à sa juridiction immédiate et canonique que ces nouvelles écoles se trouveront soustraites, 
tant au niveau diocésain qu'au niveau paroissial, et que, d'autre part, les parents des enfants catholiques et l'Eglise elle-même garderont l'obligation sacrée, en vertu même de leur foi, d'assurer la formation chrétienne et catholique de tous leurs jeunes. A remarquer ici que cette option n'est pas sans causer de graves problèmes de conscience, de grands déchirements intérieurs, chez beaucoup de ces gens droits et sincères, que l'unanimité est loin d'être faite dans le milieu même en faveur de cette nouvelle formule scolaire. A noter enfin que l'extraordinaire nouveauté ici réside dans le fait, d'une part, que des évêques comme Son Excellence Mgr Johnson, archevêque de Vancouver, et Son Excellence Mgr de Roo, évêque de Victoria, semblent non seulement ne pas s'y opposer, mais même y consentir dans les circonstances actuelles et dans le fait, d'autre part, que le ministère de l'Education de la Province semble l'accueillir avec beaucoup de sympathie et se déclarer même prêt à mettre en vigueur de nouvelles politiques scolaires qui, dans un avenir assez rapproché, permettraient de faire de ces nouvelles écoles publiques françaises une vivante et dynamique réalité.

A l'horizon de la vie canadienne-française en Colombie-Britannique, commencent à poindre des projets scolaires lourds de possibilités non seulement pour le groupe de nos congénères qui habitent cette magnifique province, mais pour tous les autres groupements français du pays, puisque si ces écoles publiques françaises voyaient enfin le jour là-bas, cela créerait un précédent "sans précédent" dans tout le Canada et que partout également, du moins en dehors du Québec, l'Eglise serait appelée à reviser ses politiques et sa pastorale scolaires par rapport à toutes les minorités canadiennes-françaises d'un bout à l'autre du pays. On le voit donc, tout ce qui se passe à l'heure actuelle en ColombieBritannique au moment où les Canadiens français doivent opter, semblet-il, entre l'école confessionnelle traditionnelle et l'école française nouvelle, revêt une importance et une signification singulières. C'est vraiment de l'Histoire qui se fait. L'avenir se trouve ici engagé pour les Canadiens français, pour l'Eglise et pour l'Etat canadien tout entier, tant au plan fédéral qu'au plan provincial.

\section{L'ÉCOLE CATHOLIQUE BILINGUE EN COLOMBIE-BRITANNIQUE}

De quoi s'agit-il exactement lorsque nos amis franco-colombiens projettent de nouvelles écoles qui ne soient plus d'abord privées et confessionnelles, mais publiques et françaises? Laissons-les nous l'expliquer eux-mêmes.

Ils s'en sont ouvert's avec beaucoup de netteté et de franchise dans deux documents récents d'une importance capitale pour quiconque veut comprendre le vrai problème scolaire qui se pose à eux : $1^{\circ}$ Le mémoire de la Fédération canadienne-française de la Colombie-Britannique à la Commission royale d'Enquête sur le Bilinguisme et le Biculturalisme, présenté officiellement en français à la Commission le 11 octobre 1964 et traduit en anglais peu après; $2^{\circ}$ Brief submitted by the FrenchCanadian Federation of British Columbia to the Honourable Leslie 
Peterson, Minister of Education for the Province of British Columbia on November 29, 1965, dont la traduction a paru depuis dans l'hebdomadaire La Survivance d'Edmonton, Alberta, édition du 9 mars 1966. Les deux documents se complètent admirablement l'un l'autre. Il faudrait les citer en entier, ce qui serait trop long. Comme ils se recoupent assez fréquemment, nous irons de l'un à l'autre au besoin et prendrons ici ou là la formule qui semble le mieux exprimer l'idée ou le fait à faire valoir.

A la racine du problème il y a, semble-t-il, l'état de fait suivant :

* Bien que la Colombie-Britannique ait eu une population de langue française d'importance variable pour plus de cent ans, rien, dans ses statuts, n'indique des précédents qui reconnaissent ce fait. Néanmoins, 66,000 Colombiens ont déclaré leur origine française au recensement de 1961. De ceux-ci 26,000 ont déclaré le français comme étant leur langue maternelle. De plus, 55,000 se réclament d'être bilingues. Pas une école officiellement reconnue par le Département de l'Education, n'a jamais été mise à la disposition de ceux qui pouvaient avoir désiré que le français soit utilisé comme langue d'enseignement. Il nous semble que tout effort en vue d'élaborer un programme scolaire reflétant la dualité canadienne, même s'il ne se traduisait, au départ, que par la création d'écoles pilotes dans les centres où le nombre de francophones peut justifier une telle initiative, serait de grande importance dans la recherche d'un consensus national.

* En principe, l'éducation relève du droit strict des parents. Bien que toute l'évolution des structures de l'enseignement doive avoir à l'esprit ce droit sacré, notre société humaine est devenue trop complexe et les exigences de notre civilisation technocratique trop lourdes à porter pour considérer, dorénavant, la primauté de l'initiative privée en matière d'instruction.

* En pratique, la socialisation de l'instruction a forcé une standardisation des programmes et la recherche d'un dénominateur commun pour tous. Ce dénominateur commun est l'Etat.

« Dans le passé, des groupes de parents, soit par leur Eglise, soit par l'intermédiaire de conseils élus, pouvaient' prélever suffisamment de fonds pour construire et administrer des écoles pour l'instruction de leurs enfants. A l'exception de quelques Eglises en Colombie-Britannique qui cherchent encore des solutions à la préservation de l'école indépendante, ce système a été abandonné.

«Pour le citoyen de langue française de la Colombie-Britannique, la situation est encore plus désastreuse. Rien n'a été prévu, au point de vue programme ou dans les cadres de l'instruction publique, pour qu'il puisse jouir d'un programme d'enseignement conforme à son identité historique canadienne. Les Canadiens d'expression française ont dû se regrouper dans de rares institutions paroissiales qui, malgré leurs efforts, ont été paralysées à cause de circonstances faciles à comprendre.

"Ces circonstances sont les suivantes : 
(1) Ces écoles, malgré leur caractère privé, du point de vue de la loi, ont dû « se soumettre au programme et à toutes les autres directives du ministère de l'Education de la province de la Colombie-Britannique ") (Règlements du $3^{\circ}$ synode de l'Archidiocèse de Vancouver, 1959, article 502, par. 2).

(2) L'enseignement du français, dans ces écoles, n'est que marginal à cause des exigences du programme officiel.

(13) Les parents n'ont aucun droit de recours au produit de leurs impôts pour l'instruction de leurs enfants.

(4) Ils ne peuvent obtenir aucun octroi et aucune compensation pour le choix d'une école plus conforme aux aspirations légitimes des parents.

"Nous pouvons tirer une conclusion générale de ces faits. En tant que citoyen et contribuable, le Canadien français doit se tourner vers son gouvernement et tenter d'inscrire ses besoins dans les cadres de la loi ${ }^{1}$.

Un peu plus loin, dans le même mémoire présenté au ministère de l'Education de la Colombie-Britannique, l'on trouve une analyse succincte, mais combien lucide et précise, de la situation des écoles déjà existantes :

" Au point où en sont les choses, les seules écoles, que nous pouvons appeler nôtres - même s'il y a ici exagération puisque, même si nous sommes appelés à leur donner notre support, elles sont, de fait, la propriété de l'Archevêque catholique de Vancouver - sont : l'école Notre-Dame-de-Lourdes et l'école Notre-Dame-de-Fatima, à Maillardville, et l'école Saint-Sacrement, rue Heather, à Vancouver.

" Dans ces écoles, même si le programme officiel est en vigueur, le français est enseigné durant de courtes périodes à partir de la première année et du jardin d'enfants. Les maîtresses sont qualifiées pour enseigner dans les deux langues. Dans la plupart des cas, leur langue maternelle est le français. De grands sacrifices ont été consentis, tant de la part des parents que des institutrices, religieuses et laïques, pour maintenir ces écoles ${ }^{2}$.

Malgré tous ces sacrifices, à quel résultat a-t-on abouti? A ceci que rapporte l'autre mémoire, présenté à la Commission LaurendeauDunton :

* A cause de l'intransigeance de la loi scolaire de la province de la Colombie-Britannique, ces écoles doivent se plier aux exigences du programme imposé et donner au compte-gouttes les éléments de la langue maternelle, matière qui n'assure aucun avantage particulier, plus tard, dans les écoles plus avancées.

* La population scolaire de ces trois écoles est d'environ 1,250 enfants. Les moyens financiers sont insuffisants pour pouvoir permettre

1 Mémoire présenté au ministère de l'Education de la Colombie-Britannique par la Fédération canadienne-française de la Colombie-Britannique, le 29 novembre 1965. Traduction française parue dans La Survivance d'Edmonton, Alberta, le 9 mars 1966, page 7 , numéros 14-22.

2 lbid., nos 27-28. 
la poursuite des cours jusqu'au brevet de $12^{\circ}$; ce qui veut dire que la plupart des enfants sont obligés de choisir une autre école après la $7^{\circ}$.

Ce sont des écoles tolérées par le ministère de l'Education à titre d'institutions privées, ne pouvant se réclamer d'aucun statut légal et, par conséquent, n'ayant pas droit au partage des revenus de la taxation scolaire. Les contribuables qui favorisent l'école "confessionnelle» ou " privée » doivent en assurer tous les frais et sont obligés, de par la loi, de se soumettre, en plus, à l'imposition dont bénéficie seule l'école publique. C'est le régime communément appelé de la « double taxation ${ }^{3}$,.

De plus, il faut bien le dire, la cause du français qui s'est jusqu'à maintenant identifiée avec la cause de l'école catholique en s'y subordonnant, a subi une double défaite, en Colombie-Britannique comme ailleurs. * Défaite vis à vis de l'Etat parce que le dialogue s'est orienté presque entièrement vers la reconnaissance officielle de l'école * séparée s confessionnelle, en dehors de toutes considérations linguistiques. Défaite à l'intérieur même du contexte des écoles dites \& privées * ou " séparées *, partout ou l'élément de langue française catholique s'est trouvé minoritaire ${ }^{4}$.

Il est évident dès lors que si les choses continuent d'aller comme elles vont, " à moins d'une certaine reconnaissance officielle de l'Etat s, laquelle ne viendra que si l'école chez les Canadiens français de la Colombie effectue un nouveau départ, "la minorité de langue française, en Colombie-Britannique, malgré tous les sacrifices qu'elle s'est imposés n'a aucune chance de se maintenir ". "Les élèves des trois écoles pseudo-françaises de la région de Vancouver doivent choisir une autre école avant d'atteindre les grades du High School ${ }^{5}$. , Et l'on aboutit ainsi à l'incroyable anomalie suivante :

* Les élèves sortant de nos écoles destinées à des enfants dont la langue maternelle est la française doivent être préparés à s'intégrer à d'autres écoles avant que la période normale d'entraînement primaire ne soit terminée. Les titulaires sont forcés de donner une part insuffsante à l'instruction française. En somme, ces écoles sont en directe concurrence avec l'école publique; condition qui n'a d'autre échéance logique que la disparition du français à l'intérieur même des écoles qui ont voulu se donner mission de le perpétuer ${ }^{6}$ !" Tableau sombre évidemment, peut-être un peu trop chargé. Mais il faut comprendre que les auteurs avaient à le brosser devant une commission du gouvernement fédéral et qu'il s'agissait d'alerter celle-ci aussi vivement que possible devant une situation qui demeure infiniment tragique...

3 Mémoire de la Fédération canadienne-française de la Colombie-Britannique présenté à la Commission royale d'Enquête sur le Bilinguisme et le Biculturalisme au Canada, le 11 octobre 1964, numéros 10, 11, et passim 16, 17, 18.

4 Ibid., no 29.

5 Ibid., no 37 .

B Ibid., no 38. 


\section{VERS L'ÉTABLISSEMENT D'ÉCOLES FRANÇAISES}

Devant une telle situation et qui ne peut qu'empirer si rien ne change, l'on comprend que lors de son dernier congrès tenu à Maillardville les 9,10 et 11 octobre 1965, la Fédération canadienne-française de la Colombie ait adopté la résolution suivante : "Que le Bureau de direction soit autorisé à approcher les autorités du ministère de l'Education aussitôt que possible en vue d'obtenir des écoles françaises dirigées et administrées par des commissaires de langue française élus par les

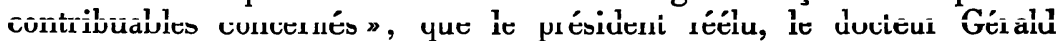
Moreau, dans un message paru au lendemain de ce congrès, dans la Survivance du 3 novembre 1965, ait établi dans son programme d'action, une échelle de priorités sur les deux prochaines années. Cette échelle de priorités est la suivante :

I Extension du réseau français de radio et de télévision à la Colombie pour refranciser notre population.

II Obtention d'écoles françaises pour freiner l'anglicisation des $\mathrm{Ca}$ nadiens français.

III Trouver le plus tôt possible le moyen positif et définitif d'assurer la viabilité du secrétariat.

Dès le 29 du même mois de novembre, le docteur Moreau, accompagné de M. Roméo Paquette, agent de liaison de la Fédération, allait présenter le mémoire que l'on sait au ministre de l'Education, geste posé au nom de la Fédération entière. Ils déclaraient' sans ambages au ministre qu'un de leurs principaux problèmes était celui de rendre les trois écoles décrites plus haut, acceptables, du point de vue administratif, par le ministre de l'Education. En même temps, ajoutaient-ils, " nous ne pouvons pas oublier les agglomérations de langue française, en Colombie, qui n'ont recours à aucune école dite bilingue ». De ces deux situations, il faudrait absolument tenir compte, a celle des écoles en existence et celle de l'absence de toute école ${ }^{7}$ ".

* Là où des écoles paroissiales existent :

(a) Un conseil composé de membres parlant français, nommés après consultation entre notre exécutif et le département, pourrait être créé dans le but d'examiner les moyens appropriés pour louer, si possible, des locaux dans les écoles existantes et pour utiliser ceux-ci aux fins d'un programme qui se concrétiserait graduellement.

( b) Dû au fait qu'un tel programme devrait débuter à la première année et qu'une année additionnelle devrait s'ajouter à chaque année subséquente, les locaux loués dans ces écoles suffiraient pour les deux ou trois premières années. S'il peut s'avérer convenable et pratique éventuellement, de convertir toute l'école en une école fondamentalement française, des ententes pourraient être négociées en vue de louer tout l'édifice.

«c) Même si l'on trouve opportun d'engager le personnel actuel de ces écoles, ou de la partie de l'école affectée, ce personnel ne serait plus employé par les autorités paroissiales, ni dirigé par elles, et les locaux ne feraient plus partie des écoles catholiques. Du même coup, même si le personnel et la majorité des élèves pourront être de foi catholique, la religion, comme telle, ne serait plus un sujet du programme officiel.

7 Mémoire au ministère de l'Education, nos 29, 30. 
(1) Ces locaux, appelés par convenance classes pour les étudiants de langue française, pourraient être mis à la disposition de quiconque préfèrerait ce mode d'instruction, dans le but de maîtriser la langue française, sans discrimination 8 .

* Et là où il n'y a pas d'école :

( Un conseil, composé de parlants français, nommé après consultation entre notre exécutif et le département, pourrait être créé en vue d'entamer des négociations avec le surintendant d'un district scolaire donné, dans le but d'utiliser une partie des locaux scolaires disponibles ou de construire une école dans le district scolaire pour les éléments de langue française qui choisiraient d'envoyer leurs enfants à une école dont la langue d'enseignement serait le français ${ }^{9}$. 》

Le mémoire continue en abordant les sujets très importants de la composition du programme scolaire, du financement, de l'administration, de l'embauchement des titulaires, d'échange d'instituteurs avec la province de Québec ou avec d'autres provinces. Remarquez bien que ce que ces chefs veulent ce n'est pas une école « bilingue » à parts égales pour le français et pour l'anglais, ni une école bilingue qui leur serait accordée en vertu d'une sorte de privilège, ni une école bilingue qui serait le fruit de demi-mesures pour autant que le français y serait impliqué, mais bien une école publique authentiquement française, adaptée aux vrais besoins de Canadiens français vivant en ColombieBritannique à l'intérieur d'une Confédération renouvelée, à partir d'un bilinguisme et d'un biculturalisme anglais et français acceptés dans les structures scolaires des provinces elles-mêmes et sanctionnés par la loi.

\section{CONCLUSION}

Ce projet a-t-il des chances de réussir, avec les modifications inévitables qu'une étude plus approfondie pourrait apporter sur un point ou sur un autre, l'essentiel étant sauvegardé?

Il semble bien que oui. Le ministre de l'Education, en deux rencontres subséquentes, n'a cessé de l'accueillir avec beaucoup d'intérêt et de sympathie. Les autorités ecclésiastiques, loin de se montrer hostiles, reçoivent et encouragent ceux qu'ils savent être les initiateurs et les protagonistes du projet lui-même. L'Eglise, là-bas comme ailleurs, devrait être capable de rajuster sa pastorale en matière d'éducation selon les situations nouvelles qu'elle rencontre sur sa route en prenant soin du peuple de Dieu dans le monde d'aujourd'hui. Enfin, les hommes qui travaillent à l'avènement de ce régime scolaire nouveau comprennent parfaitement l'enjeu du changement. Ils ne veulent rien sacrifier des valeurs ici impliquées, valeurs catholiques et valeurs françaises. Leur grand souci, au contraire, est de les assurer toutes deux à une population

$8 \quad$ Ibid., no 31.

$9 \quad$ Ibid., no 32. 
qui, dans les cadres des institutions scolaires actuelles, est menacée de perdre ses valeurs françaises et, par conséquent, la moitié de son âme ${ }^{10}$.

Il me semble qu'en pareille conjoncture, on ne peut que souhaiter qu'ils réussissent et que leurs amis, de près et de loin, s'emploient à les aider dans l'œuvre admirable, difficile, nécessaire et urgente qu'ils poursuivent.

\author{
11 juin $1966 . \quad$ Thomas-M. LANDRY, o.p. \\ Directeur des Relations extérieures \\ Conseil de la Vie Française en Amérique
}

10 Consulter aussi à ce sujet: L'Appel, magazine des Franco-Colombiens publié mensuellement par la Fédération canadienne-française de la Colombie, 333, rue Walker, Maillardville, C.B.; L'Echo de la Colombie, organe officiel du Club canadien-français de Victoria, 1660, Ruby Road, Victoria, C.B., bulletin mensuel; La Survivance, Edmonton, Alberta une page entière consacrée chaque mois à la vie canadienne-française en Colombie-britannique; Conseil de la Vie française en Amérique, 75, rue d'Auteuil, Québec-4, P.Q. : archives de la Colombie-britannique.

Afin de connaître la toile de fond, l'arrière-plan historique, consulter : les œuvres classiques du R.P. Morice, o.m.i. et du chanoine Lionel Groulx et, tout récemment, Mason Wade, dans Canadian Dualism - La Dualité canadienne, 1960; The University of British Columbia, Vancouver, Canada, The Journal of Education of the Faculty of Education, Vancouver, number 9, January 1964, Special issue on Biculturalism and Education; Commission royale d'Enquête sur le Bilinguisme et le Biculturalisme au Canada, Rapport intérimaire, paru le ler février 1965; Paul-Emile Gosselin, P.D., L'Empire français d'Amérique, Les Editions Ferland, 75, rue d'Auteuil, Québec-4, P.Q., 1963; Conseil de la Vie Française en Amérique, Mémoire présenté en 1964 à la Commission royale d'Enquête Laurendeau-Dunton; (Le Bilinguisme et le Biculturalisme au Canada »: autre mémoire présenté en 1965 au Comité Parlementaire de la Constitution du gouvernement de la Province de Québec, «L'Avenir du Peuple canadien-français », parus tous deux aux Editions Ferland de Québec. 